\title{
A TRANSIÇÃO DA MORTALIDADE POR CÂNCERES NO BRASIL E A TOMADA DE DECISÃO ESTRATÉGICA NAS POLÍTICAS PÚBLICAS DE SAÚDE DA MULHER
}

\author{
Raphael Mendonça Guimarães \\ Fundação Oswaldo Cruz (FIOCRUZ) \\ Camila Drumond Muzi \\ Instituto Nacional de Câncer (INCA) \\ Moema de Poli Teixeira
Fundação Cesgranrio \\ Sonoe Sugahara Pinheiro \\ Instituto Brasileiro de Geografia e Estatística (IBGE)
}

\section{A TRANSIÇÃO DA MORTALIDADE POR CÂNCERES NO BRASIL E A TOMADA DE DECISÃO ESTRATÉGICA NAS POLÍTICAS PÚBLICAS DE SAÚDE DA MULHER}

Resumo: Este artigo tem por objetivo descrever a evolução da transição da mortalidade por cânceres de mama e colo do útero no Brasil em relação ao desenvolvimento socioeconômico. Foram obtidos dados de mortalidade por câncer de mama e de colo do útero no Brasil para um período de 20 anos, correspondente a um intervalo bicensitário. Os óbitos foram corrigidos e as taxas padronizadas. Além disso, indicadores sociais foram mensurados para os três anos censitários incluídos na série temporal. Observa que os estados brasileiros possuem muita heterogeneidade com relação aos indicadores sociais e à mortalidade pelos dois cânceres, e parece haver um padrão entre a condição socioeconômica e a mortalidade por câncer de mama e de colo do útero. Conclui que é necessário, portanto, realizar a avaliação e o planejamento das ações, considerando cenários de desenvolvimento, para garantir o acesso universal e equitativo das mulheres às políticas públicas de saúde.

Palavras-chave: Demografia, Mortalidade, Desenvolvimento, Câncer, Políticas públicas, Políticas de saúde.

\section{CANCER'S TRANSITION IN BRAZIL AND STRATEGICAL DECISION-MAKING IN WOMEN'S PUBLIC HEALTH POLICIES}

Abstract: This article aims to describe the evolution of the transition in mortality from breast and cervix cancers in Brazil in relation to socioeconomic development. There were data obtained of mortality from breast cancer and cervical cancer in Brazil for a period of 20 years, corresponding to a censitary range. Deaths were corrected and rates standardized. In addition, social indicators were measured for all three census years included in the time series. It was observed that the Brazilian states have a lot of heterogeneity with respect to social indicators and mortality for both cancers, and there seems to be a pattern between socioeconomic status and mortality from breast and cervix cancer. It is therefore necessary to carry out the assessment and planning of actions, considering development scenarios, to ensure universal and equal access of women to public health policies.

Key words: Demographics, Mortality, Development, Cancer, Public policies, Health policies. 
1 INTRODUÇÃO

É crescente a carga de doenças crônicas não transmissíveis em todo o mundo, e muito se fala a respeito de uma ação global para conter esta crescente carga, especialmente nos países de baixa e média renda, uma vez que este grupo de doenças representa atualmente $80 \%$ da carga de doença mundial (FARMER; FRENK; KNAUL, 2010).

Em particular, a previsão é de que haja aproximadamente 13,2 milhões de óbitos por câncer em todo o mundo em 2030, um número consideravelmente maior que os 7,6 milhões de óbitos em 2008 (FERLAY et al., 2010).

Bray e outros (2012) avaliaram os padrões mundiais de carga de câncer, tanto em termos de incidência e mortalidade, e previram cenários futuros em relação aos diferentes níveis de desenvolvimento socioeconômicos, medidos usando o Índice de Desenvolvimento Humano (IDH). Este estudo fornece uma boa explicação da teoria da transição do câncer, que serve para fins de pesquisa e orientação na definição de prioridades para o controle do câncer.

A transição do câncer pode ser considerada como uma extensão ou a conclusão da teoria de Omran da transição epidemiológica. No princípio desta teoria se observa uma mudança de doenças infecciosas para as doenças não transmissíveis, e neste sentido a teoria da transição do câncer vê uma mudança da predominância dos casos de cânceres ligados a infecções para os cânceres associados a fatores de risco que são principalmente não-infecciosos e possivelmente relacionados a um estilo de vida chamado ocidental. Assim, Clegg, Reichman e Miller (2009) apontam que, em áreas de alto IDH na Europa, quatro tipos de câncer (pulmão, mama feminina, cólon e reto e próstata) são responsáveis por quase a metade da carga total de incidência de câncer; nas áreas de IDH médio, misturam-se altas taxas de câncer de pulmão, cólon e reto, estômago e fígado; finalmente, em áreas com baixa IDH, os tipos de câncer mais frequentes parecem ser os do colo do útero, o fígado, o sarcoma de Kaposi e linfoma de não-Hodgkin, todos tipicamente com origem em agentes infecciosos (respectivamente, HPV, vírus da hepatite B e C e HIV).

Nos estudos de Bray e outros (2012), Clegg, Reichman e Miller (2009) e Ward e outros (2004), todos explorando 0 contexto do desenvolvimento para explicar a dinâmica de incidência e mortalidade por tipos de cânceres específicos, fica clara a associação que o padrão de morbimortalidade por este grupo de causas tem relação direta com o contexto socioeconômico.

$$
\text { Emblematicamente, }
$$
as disparidades sociais fazem parte do escopo de discussão da Política Nacional de Atenção Integral à Saúde da Mulher (PNAISM). Com o 
avanço dos debates em torno dos direitos das mulheres, a PNAISM também passou a considerar a desigualdade de gênero como fator de grande impacto sobre as condições da saúde da mulher e que, portanto, precisa ser considerada, tanto na análise das ações no âmbito do Sistema Único de Saúde (SUS) como dentro das diretrizes e princípios estabelecidos pelo Ministério da Saúde (MS) para esta parcela da população. Desta forma, a agenda da área técnica de saúde da mulher passou a incorporar aspectos relevantes da saúde da população feminina, tais como a assistência às doenças ginecológicas prevalentes, a prevenção, a detecção e 0 tratamento do câncer de colo uterino e de mama (BRASIL, 2014). Estas duas topografias de cânceres ginecológicos, de maior magnitude em todo o mundo, possuem ocorrência heterogênea entre os países. 0 padrão de ocorrência destes cânceres, seja para a incidência ou para a mortalidade, mostra que 0 câncer do colo do útero é mais expressivo que o câncer de mama para países de renda baixa e média-baixa, e o oposto para países de renda alta e média-alta. De fato, aproximadamente $80 \%$ dos casos de câncer do colo do útero ocorrem em países em desenvolvimento (sendo, inclusive, o câncer mais incidente entre mulheres nestas localidades). Suas maiores taxas se apresentam na América Latina, Caribe, África Subsaariana e Sudeste Asiático. Já o câncer de mama possui maior magnitude em países desenvolvidos e em transição de desenvolvimento. Em geral, é citado como sendo consequência da ocidentalização do mundo, através do aumento da prevalência de hábitos tipicamente ocidentais (como 0 excesso e consumo de álcool, fatores dietéticos e inatividade física) (FERLAY et al., 2015).

Em estudo recente que analisou a evolução da mortalidade por câncer do colo uterino e de mama no Brasil, segundo indicadores socioeconômicos e assistenciais, observou-se queda da mortalidade por câncer do colo uterino em todo o período, exceto em municípios das regiões Norte e Nordeste fora das capitais. Houve, para o mesmo período, declínio na mortalidade por câncer de mama nas capitais a partir do final da década de 1990. Os indicadores socioeconômicos positivos correlacionaram-se inversamente com a mortalidade de câncer do colo uterino. Ainda, observou-se forte correlação direta entre indicadores positivos e a mortalidade por câncer de mama nos municípios do interior dos estados. Acredita-se que estas associações se deem num contexto de aumento de risco por câncer de mama e do colo uterino com atenuação da mortalidade em função da expansão de oferta e acesso ao rastreamento, diagnóstico e tratamento, porém de forma desigual (AZEVEDO E SILVA et al., 2014).

Sendo assim, este artigo tem por objetivo descrever a evolução da transição da mortalidade por cânceres femininos (mama e 
colo do útero), no Brasil, em relação ao desenvolvimento humano, identificando diferenças regionais para a avaliação das políticas públicas de saúde da mulher.

\section{MATERIAL E MÉTODOS}

A análise foi realizada a partir de dados agregados por estados brasileiros entre os anos de 1991 e 2010, correspondendo a um intervalo de 20 anos, cujo início e término se dão em anos censitários. Isto permitiu a obtenção, além dos dados de mortalidade para todos os anos, a informação sobre 0 desenvolvimento humano e demais indicadores socioeconômicos utilizados.

Os dados de óbitos foram extraídos do Sistema de Informação sobre Mortalidade (SIM). Para o cálculo das taxas, foram obtidos dados populacionais dos censos demográficos do Instituto Brasileiro de Geografia e Estatística (IBGE) e os indicadores socioeconômicos e assistenciais do Instituto de Pesquisa Econômica e Aplicada (IPEA).

Para os óbitos entre 1991 e 1995, foram considerados os dados referentes à codificação do Manual da Classificação Estatística Internacional de Doenças, Lesões e Causas de óbito (CID-9); enquanto os de 1996 a 2010, a partir da Classificação Estatística Internacional de Doenças e Problemas Relacionados à Saúde (CID-10). Os cânceres de mama e de colo do útero correspondem, respectivamente, aos códigos: 174 (CID-9) e
C50 (CID-10), 180 (CID-9) e C53 (CID-10), informados pelo SIM. Foi realizada correção dos óbitos por câncer de mama e de colo do útero, empregando a metodologia de redistribuição proporcional utilizada pela Organização Mundial da Saúde (OMS). As taxas foram padronizadas pelo método direto, utilizando como população padrão a população mundial estimada por Segi e outros (1960) e adaptada por Doll, Payne e Waterhouse (1966).

Já os indicadores socioeconômicos foram obtidos para anos censitários. O coeficiente de Gini (grau de concentração da distribuição de renda domiciliar per capita de uma determinada população) e a razão de renda (Número de vezes que a renda agregada do quinto superior da distribuição da renda ( $20 \%$ mais ricos) é maior do que a renda do quinto inferior $(20 \%$ mais pobres)) foram utilizados para medir a desigualdade de distribuição de renda. Já 0 grau de urbanização (percentual da população residente em áreas urbanas, em determinado espaço geográfico) foi utilizado por acompanhar o processo de urbanização da população brasileira, em diferentes espaços geográficos, subsidiando processos de planejamento, gestão e avaliação de políticas públicas, para adequação e funcionamento da rede de serviços sociais e da infraestrutura urbana, servindo, portanto, como uma proxy de oferta de serviços (inclusive de saúde). 
Finalmente, o IDH engloba, além da componente renda, os componentes longevidade e educação. Para fins de análise, os estados foram divididos nos quartis gerados pelos valores dos IDH.

\section{RESULTADOS}

Há um avanço geral dos estados brasileiros com relação aos indicadores sociais. Ao analisar os indicadores negativos, ou seja, a razão de renda (Gráfico 1) e o índice de Gini, que avaliam a desigualdade social (Gráfico 2), entretanto, vemos que esta evolução ainda é acompanhada de alguma disparidade. A razão de renda, ao longo dos 20 anos analisados, caiu no Brasil em todos os estados, e houve ainda uma redução na disparidade entre as taxas dos estados. Já o Índice de Gini, embora haja uma grande variação ao longo dos 20 anos analisados (o que fica evidente com a oscilação dos países que compõem o grupo

\section{Gráfico 1 - Evolução da razão de renda em estados brasileiros. Brasil, 1991-2010}

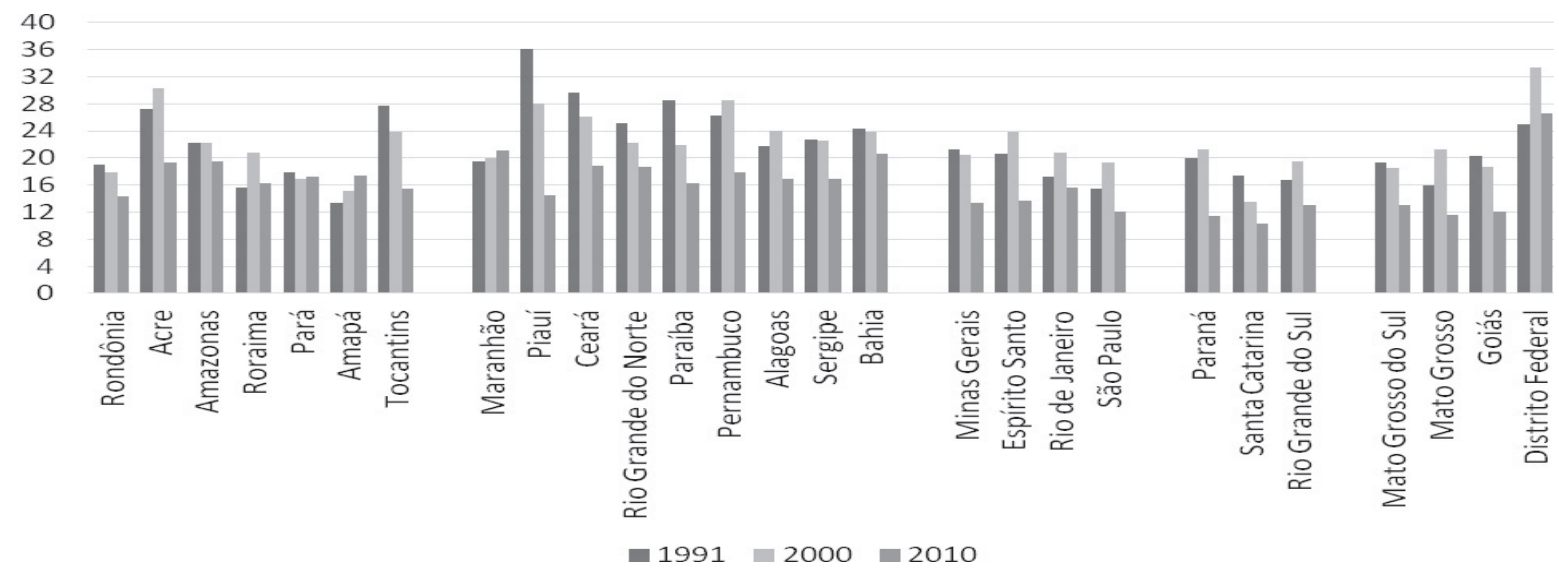

Fonte: INSTITUTO BRASILEIRO DE GEOGRAFIA E ESTATISTICA. Estimativas populacionais para os municípios e Unidades da Federação brasileiros. Rio de Janeiro, 2015. Disponível em:<http://www.ibge.gov.br/home/estatistica/populacao/estimativa2015/default.shtm>. Acesso em: 2 jan. 2016.

\section{Gráfico 2 - Evolução do Índice de Gini em estados brasileiros. Brasil, 1991-2010}

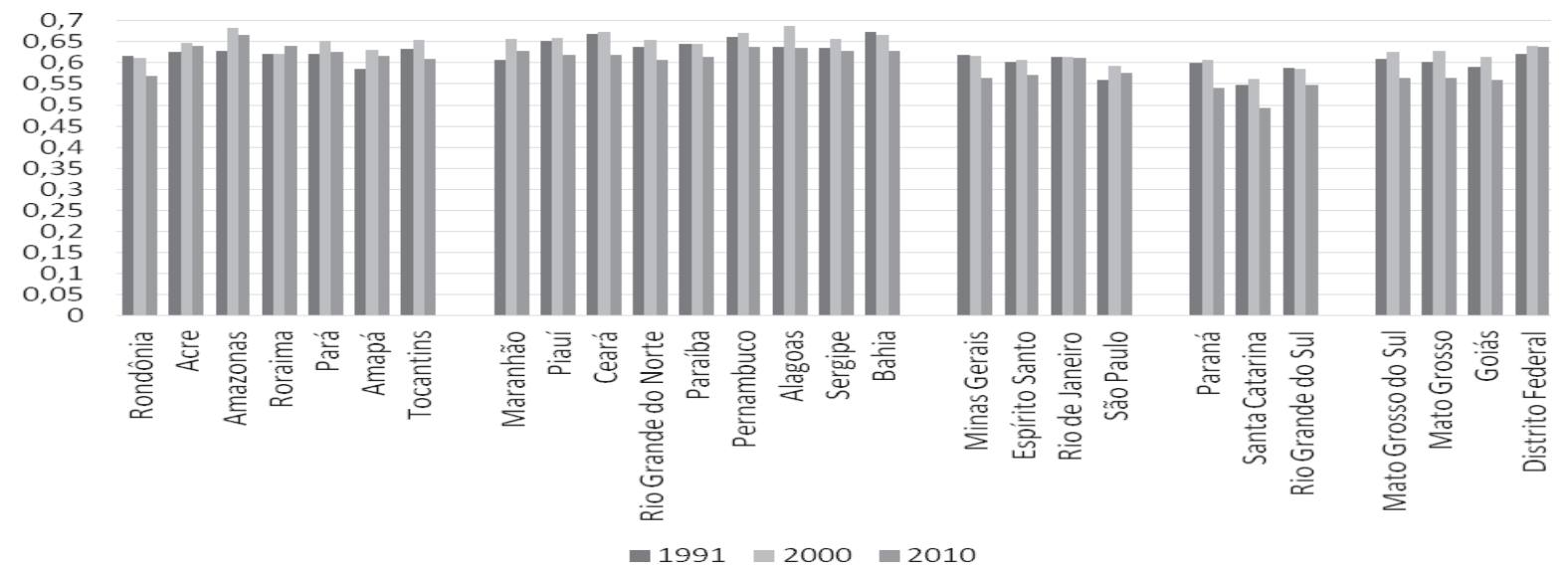

Fonte: (INSTITUTO BRASILEIRO DE GEOGRAFIA E ESTATISTICA, 2015). 
com os 5 maiores e menores valores de taxas), aumentou ao longo dos anos, fazendo com que os estados com maiores desigualdades fossem se distanciando daqueles onde a desigualdade parece ter diminuído ou se estabilizado.

Ao se avaliar os indicadores positivos, ou seja, o grau de urbanização (Gráfico 3) e o IDH (Gráfico 4), observa-se a melhora dos indicadores nos estados. De uma forma geral, houve um crescimento do grau de urbanização de todos os estados, sem variação dos 5 estados com maior renda média e dos 5 com menor renda. Houve, ainda, uma redução na disparidade entre os estados. Finalmente, a avaliação do IDH mostra que houve um crescimento global do desenvolvimento humano nos estados, com redução das disparidades entre eles. Constatou-se a formação de aglomerados espaciais de com IDH semelhantes. $O$ grupo de IDH muito baixo agrega quase a totalidade dos estados do Nordeste, à exceção do Tocantins e Rondônia,

Gráfico 3 - Evolução do grau de urbanização em estados brasileiros. Brasil, 1991-2010
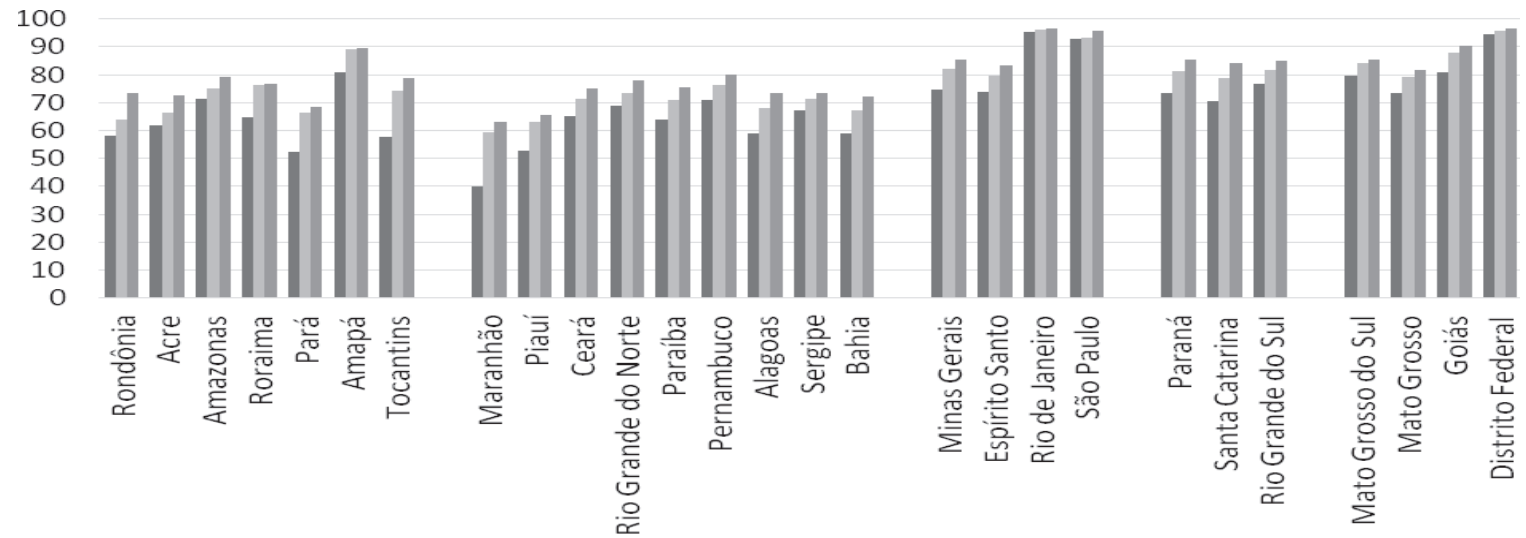

$$
\text { - } 1991 \square 2000 \quad 2010
$$

Fonte: (INSTITUTO BRASILEIRO DE GEOGRAFIA E ESTATISTICA, 2015).

Gráfico 4 - Evolução do IDH em estados brasileiros. Brasil, 1991-2010

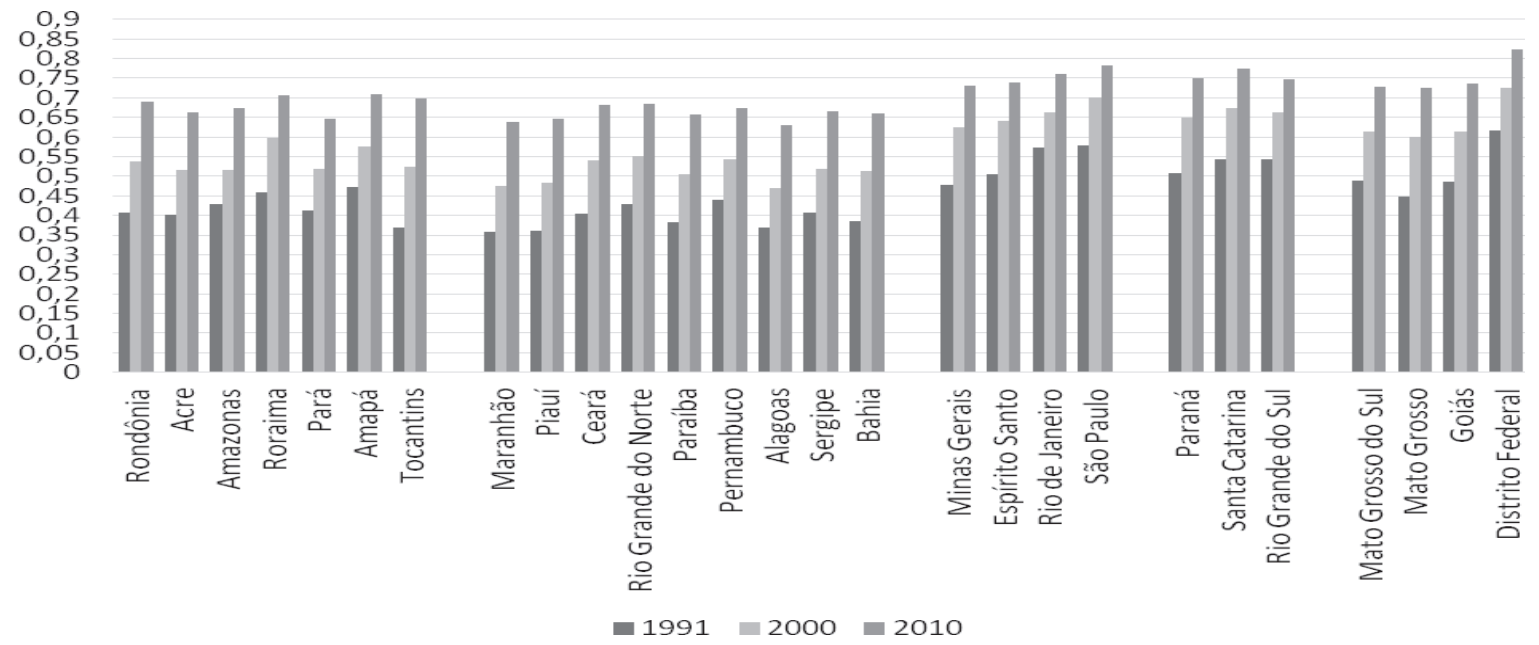

Fonte: (INSTITUTO BRASILEIRO DE GEOGRAFIA E ESTATISTICA, 2015).

R. Pol. Públ., São Luís, v. 20, n 1, p. 33-50, jan.jjun. 2016. 
ambos da região Norte. Já o grupo de baixo IDH reúne os estados do Norte e os estados do Nordeste não incluídos no grupo anterior. 0 grupo de IDH médio reúne os estados do Centro Oeste e os dois estados do Sudeste com maior variação dos indicadores sociais, seja pelo perfil do próprio estado, como é o caso do Espirito Santo, seja por agregar muitos municípios e com muitas diferenças estruturais e demográficas, como é o caso de Minas Gerais. Finalmente, o grupo com alto IDH concentra os Estados da Região Sul, Rio de Janeiro e São Paulo, evidenciando a tradicional disparidade do eixo Sul-Sudeste com 0 restante do Brasil, bem como destacando o peso que a renda possui na formulação do IDH, que é um indicador composto.

Foi ainda observada a evolução das taxas de mortalidade por câncer de mama e do colo do útero nos 3 anos censitários. Houve, para o câncer de mama, uma redução na disparidade das taxas, com um leve incremento para 2010, posição dos estados, embora houvesse uma mistura maior entre os estados do norte e nordeste no grupo com as menores taxas, e em 2010 observa-se um destaque maior dos estados no Norte neste grupo. Já para o câncer do colo do útero, a disparidade aumentou, havendo grande mobilidade na posição dos estados. Destacase, ainda, que há estados que, apesar de possuírem melhor desempenho nos indicadores sociais, permanecem com taxas de mortalidade por câncer do colo do útero intermediárias.

Ao se observar a constituição destes grupos de IDH, e a contribuição proporcional dos cânceres de mama e do colo do útero na mortalidade por cânceres femininos em mulheres em estados brasileiros (Gráficos 5 e 6), ressalta-se que no grupo de muito baixo $\mathrm{IDH}$, em geral há uma proporcionalidade maior de mortalidade por câncer do colo do útero, comparado ao câncer de mama. Como exceção, tem-se os estados da Bahia e Paraíba, em 1991 e 2000, e em 2010 as exceções crescem em número de estados incluindo-se na lista o Piauí e Alagoas, confirmando a transição nestes estados. No grupo de IDH baixo observa-se uma heterogeneidade maior entre os estados, que diminui quando observado o grupo de IDH médio; contudo a contribuição dos cânceres de mama e do colo do útero é relativamente semelhante. Ao final, o grupo de IDH alto apresenta, sem exceções, todos os estados com proporcionalidades maiores para câncer de mama, comparado ao câncer do colo do útero. Destaca-se, ainda, que estas proporções, neste grupo, são bastante díspares.

\section{DISCUSSÃO}

O controle do câncer tornou-se prioridade na Política Nacional de Atenção Oncológica (Portaria GM n. 2439, de 8 de 
Gráfico 5 - Evolução da contribuição proporcional dos cânceres de mama e do colo do útero na mortalidade por cânceres femininos em mulheres em estados brasileiros. Brasil, 1991-2010

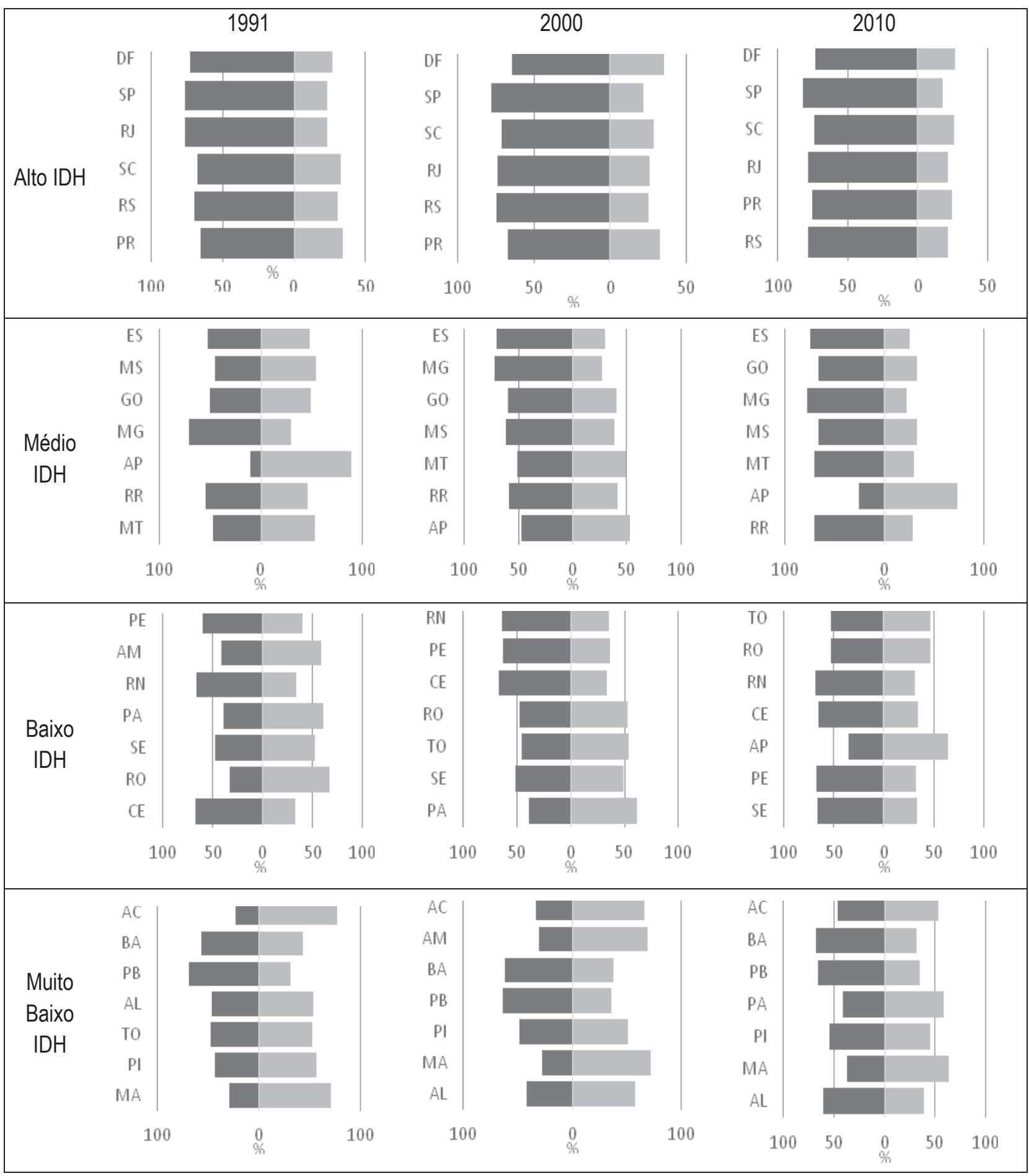

Fonte: BRASIL. Ministério da Saúde. Departamento de Informática do SUS. Sistema de Informações sobre Mortalidade. Brasília, DF, 2015. Disponível em:<http://tabnet.datasus.gov.br/cgi/deftohtm.exe?sim/cnv/obt10uf.def>. Acesso em: 2 jan. 2016.

Legenda: Mama Colo de útero. 
Gráfico 6 - Tendência temporal do IDH, da taxa de mortalidade por câncer de mama e colo do útero em estados brasileiros. Brasil, 1991-2010

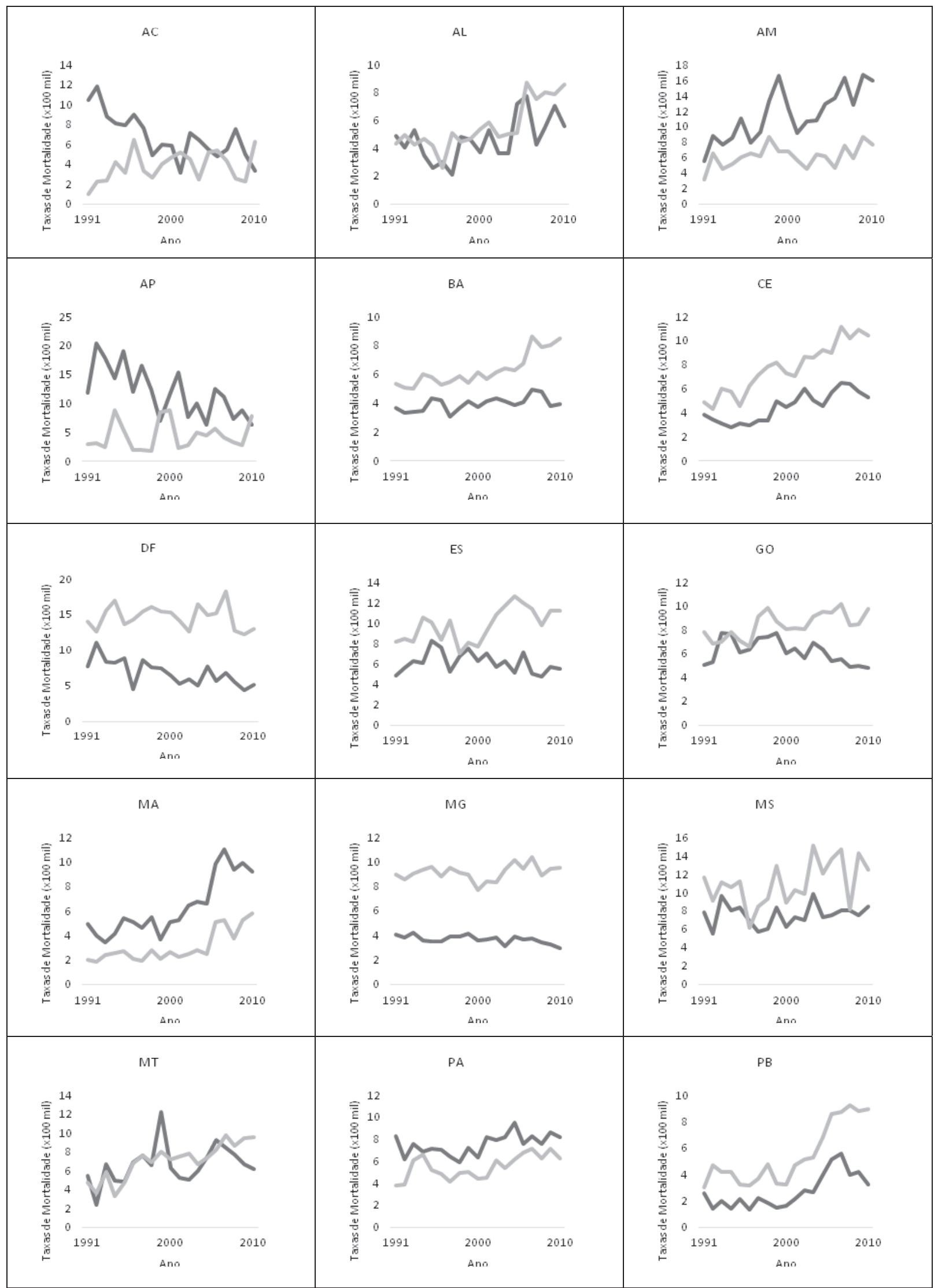




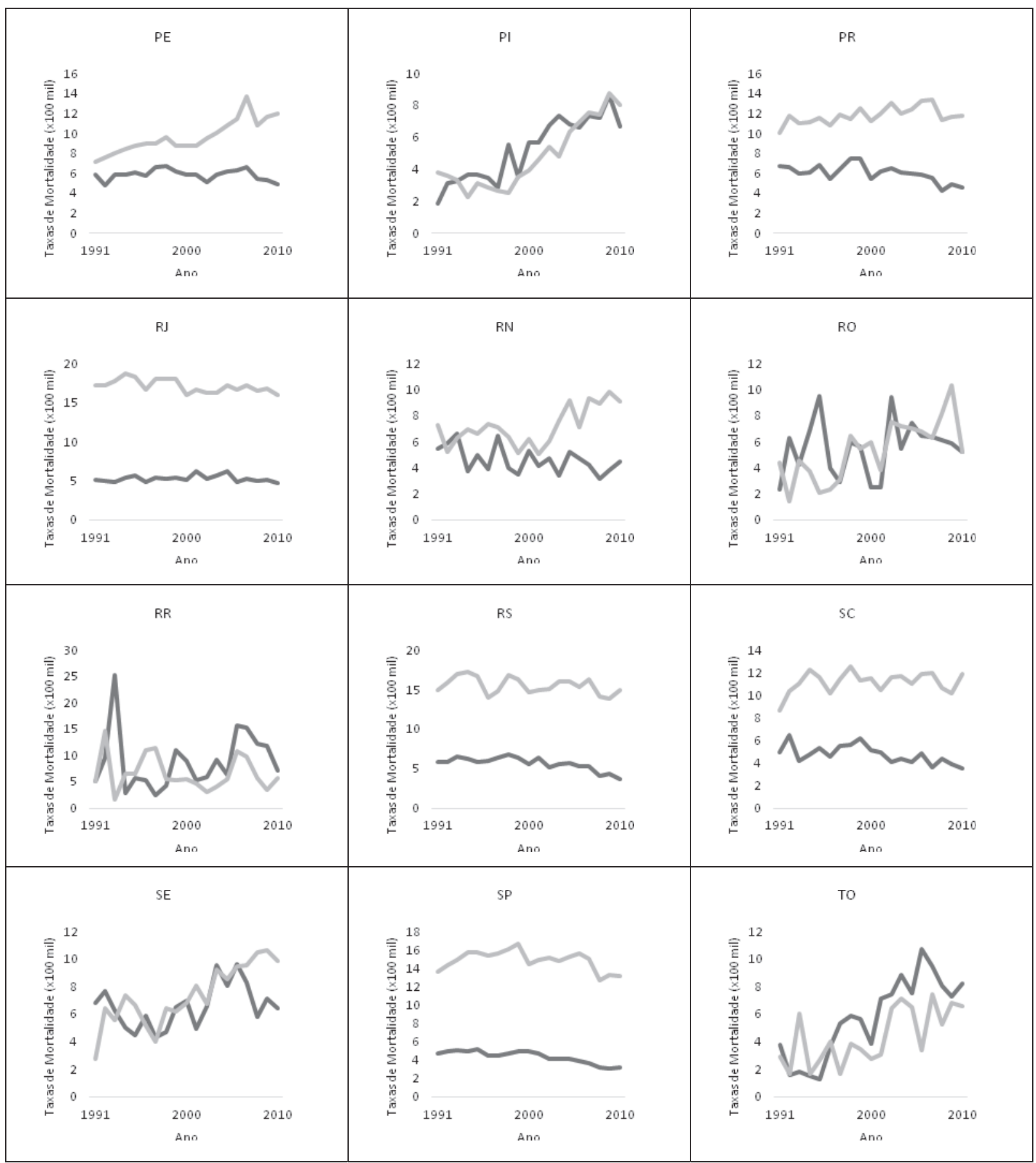

Fonte: (BRASIL, 2015).

Legenda: $\quad$ Mama Colo de útero

AC - Acre; AL - Alagoas; AM - Amazonas; AP - Amapá; BA - Bahia; CE - Ceará; DF - Distrito Federal; ES Espírito Santo; GO - Goiás; MA - Maranhão; MG - Minas Gerais; MS - Mato Grosso do Sul; MT - Mato Grosso; PA - Pará; PB - Paraíba; PE - Pernambuco; PI - Piauí; PR - Paraná; RJ - Rio de Janeiro; RN - Rio Grande do Norte; RO - Rondônia; RR - Roraima; RS - Rio Grande do Sul; SC - Santa Catarina; SE - Sergipe; SP - São Paulo; TO - Tocantins.

dezembro de 2005) e no Pacto pela Saúde, em 2006, como estratégia para tentar minimizar as taxas de mortalidade. A redução da mortalidade pelo câncer de mama feminina foi incluída como prioridade no Pacto pela Vida. Um dos objetivos firmados foi de ampliar a oferta de mamografia visando alcançar cobertura de $60 \%$ da população-alvo (INSTITUTO NACIONAL DE CÂNCER, 2015).

R. Pol. Públ., São Luís, v. 20, n 1, p. 33-50, jan.jun. 2016. 
Neste estudo, para além dos locais, o câncer do colo do útero apresentou maior mortalidade nos estratos populacionais com piores condições sociais. Em contraposição, o câncer de mama é mais expressivo nos estratos diferenciados da sociedade. Cabe ressaltar que estas tendências são dinâmicas e se transformam na medida em que se observam mudanças no perfil socioeconômico dos grupos sociais.

As taxas de mortalidade por câncer do colo do útero tendem a ser maiores em locais com IDH mais baixo. Já para 0 câncer de mama, a tendência é ser oposta, com as maiores taxas sendo observadas em locais com maior IDH. Há, entretanto, lugares com o IDH médio em que, devido à heterogeneidade da prevalência e distribuição de fatores de risco, as taxas de incidência e mortalidade por câncer de mama são relativamente altas, e as taxas de incidência e mortalidade por câncer do colo do útero não se mantém baixas.

Diversos autores encontraram uma tendência crescente de mortalidade por câncer de mama em mulheres brasileiras a partir dos 30 anos, com uma aceleração nas mulheres acima de 60 anos (FELIX et al., 2011; RODRIGUES; BUSTAMANTE-TEIXEIRA, 2011). Em um estudo realizado sobre tendência de mortalidade por câncer nas capitais e no interior do Brasil, entre 1981 e 2006, os autores relatam que a mortalidade por câncer de mama tem aumentado nos municípios do interior de todas as regiões desde a década de 1990 (SILVA et al., 2011).

O Câncer de mama apresenta maiores chances de cura quando diagnosticado precocemente. Entretanto, um estudo sobre 0 estadiamento dos casos de câncer de mama no momento do diagnóstico realizado em 89 hospitais brasileiros mostrou que somente $10,9 \%$ se encontravam no estádio I; $42,8 \%$ no estádio II; 32,6\% no estádio III e 12,3\% no estádio IV (THULER; MENDONÇA, 2005).

Em um estudo realizado em 104 pacientes no INCa, somente $25,0 \%$ das pacientes tiveram suas lesões neoplásicas detectadas por mamografia, mostrando uma inacessibilidade ao método e uma baixa oferta deste exame no serviço público local na ocasião da coleta dos dados. Contudo, quando detectados por mamografia, a maioria dos casos $(80,8 \%)$ encontrava-se nos estádios 0 ou I. (REZENDE et al., 2009).

Em Teresina, foi encontrado, em um estudo transversal realizado em 2010/2011, que $24,7 \%$ das mulheres de 40 a 69 anos nunca haviam realizado mamografia e 37,9\% não haviam realizado este exame nos últimos 2 anos (LAGES et al., 2012). Neste estudo a não realização de mamografia esteve relacionada a fatores raciais (raça parda e negra) e socioeconômicos (menor renda, menor grau de instrução e não possuir plano/seguro de saúde).

Vários estudos em países desenvolvidos detectaram uma redução na 
incidência do câncer de mama após a diminuição da prescrição de terapia de reposição com estrogênio e progesterona para mulheres na menopausa. A diminuição na prescrição destes medicamentos ocorreu após a publicação de estudos que mostraram uma relação direta entre 0 uso de terapia de reposição hormonal e o aumento do risco de desenvolvimento do câncer de mama (KATALINIC; PRITZKULEIT; WALDMANN, 2009; TORIOLA; COLDITZ, 2013; MOLINIÉ et al., 2014). O Quadro 3.1 apresenta uma seleção não sistematizada de estudos que exemplificam a tendência da incidência e mortalidade por câncer de mama.

Já para o câncer de colo do útero, a recomendação do Ministério da Saúde para rastreamento do câncer cervical uterino é a realização do exame de papanicolau, repetidos a cada três anos, após dois exames normais consecutivos realizados com um intervalo de um ano, dos 25 aos 60 anos de idade (INSTITUTO NACIONAL DE CÂNCER, 2010). Entretanto, os indicadores e suas metas são sistematicamente baixos, com marcadas desigualdades regionais no acesso à detecção precoce e à cirurgia, sendo o acesso mais baixo na Região Norte e mais alto na Região Sul (GIRIANELLI; GAMARRA; SILVA, 2014). Por exemplo, em Minas Gerais, a estimativa da razão entre exames preventivos cervicovaginais em mulheres de 25-59 anos e a população feminina nesta faixa etária, a partir de dados do Departamento de Informática do
Sistema Único de Saúde (DATASUS) no ano 2007 , foi de 0,2 . As razões acima de 0,3 podem ser consideradas adequadas de acordo com a recomendação de rastreamento pelo MS de realizar um exame a cada três anos, após dois exames normais anuais (ALVES; GUERRA; BASTOS, 2009).

\section{Thuler e Mendonça (2005)} realizaram um estudo, em duas etapas, em vários hospitais brasileiros, sobre o percentual de pacientes diagnosticadas em estádio avançado (estádios II e IV). Na primeira etapa (1990-1994), encontraram uma mediana de $56,8 \%$ de casos avançados de câncer de colo de útero. Na segunda etapa (1995-2002), foi encontrada mediana de $45,5 \%$ de casos avançados do mesmo tipo de câncer.

Um estudo nos Estados Unidos encontrou uma pequena redução na incidência de câncer de colo de útero e uma estabilização da mortalidade nos últimos cinco anos, apesar do avanço nos métodos preventivos. Relatou também que a maioria dos casos de câncer de colo de útero ocorre em mulheres que não foram submetidas a um exame preventivo recente e que ainda existem inequidades importantes no rastreamento, incidência e mortalidade. Concluiu que resolvendo os problemas que impedem a realização do rastreamento, haveria mais chances de reduzir a incidência e mortalidade por esta neoplasia (BERNARD et al., 2014).

Embora a incidência de câncer de colo de útero apresente uma tendência de 
diminuição com o aumento do desenvolvimento socioeconômico, a mortalidade pode estabilizar e continuar alta em algumas regiões caso não haja um programa adequado de rastreamento através do exame citopatológico do colo do útero e um tratamento adequado das lesões precursoras de câncer e das lesões neoplásicas em estágios precoces.

No Brasil, o rastreamento citológico para o câncer de colo de útero só começou a fazer parte das medidas de saúde coletiva em 1984, com o Programa de Assistência Integral à Saúde da Mulher (PAISM), organizado pelo MS. Com a criação do SUS, as iniciativas de combate a esta doença passaram a ser ampliadas e, em 1997, - Programa Nacional de Rastreamento do Câncer Cervical foi incorporado ao Programa Viva Mulher, priorizando mulheres entre $35 \mathrm{e}$ 49 anos (ALVES; GUERRA; BASTOS, 2009). Em 1998, o MS instituiu o Programa Nacional de Combate ao Câncer de Colo do Útero através da Portaria GM/MS n. 3040, de 21 de junho de 1998.

A redução da mortalidade pelo câncer de colo de útero também foi incluída como prioridade no Pacto pela Vida, em 2006. Os objetivos traçados foram ampliar a oferta de exame preventivo do câncer de colo de útero a fim de alcançar $80 \%$ da população alvo e tratar/seguir as lesões precursoras do câncer de colo de útero no nível ambulatorial (INSTITUTO NACIONAL DE CÂNCER, 2015). Finalmente, em 2014, o MS iniciou a imunização de meninas de 11 a 13 anos com a vacina tetravalente (HPV subtipos 6, 11, 16 e 18). Em 2015, iniciou a mesma vacinação em meninas entre 09 e 11 anos (INSTITUTO NACIONAL DE CÂNCER, 2015).

Embora a incidência de todos os tipos de câncer nos países desenvolvidos seja quase o dobro da incidência nos países em desenvolvimento, a sobrevida das pessoas com câncer é muito maior e as taxas de mortalidade são somente $21 \%$ maiores em homens e $2 \%$ maiores em mulheres nos países desenvolvidos (BRAY et al., 2011). O câncer de mama está entre os quatro tipos de câncer mais frequentes e com maior mortalidade nos países com IDH alto e muito alto, com risco cumulativo de incidência ao longo da vida maior que $3 \%$. As taxas de incidência de câncer de mama feminina vêm aumentando em quase todos os países do mundo, com elevações médias de cerca de $2 \%$ por ano. Já o câncer de colo de útero é associado negativamente ao IDH, de tal forma que é o câncer feminino com maior incidência e mortalidade em vários países com IDH baixo e médio (JEMAL et al., 2010).

A incidência de câncer de mama é maior nas regiões mais desenvolvidas, como América do Norte, o norte e oeste da Europa, Austrália e Nova Zelândia. Enquanto regiões menos desenvolvidas apresentam as menores taxas de incidência de câncer de mama, como ocorre na Ásia e partes da África. A América do Sul, o Caribe e o Norte da África apresentam 
incidência intermediária. Vários países desenvolvidos vêm conseguindo reduzir as suas taxas de mortalidade por câncer de mama, apesar do aumento da incidência mundialmente. Isto ocorreu devido à ampliação da realização da mamografia de rastreamento, diagnósticos precoces e à evolução no tratamento desta neoplasia nas últimas décadas (JEMAL et al., 2011).

Por outro lado, o câncer de colo de útero é a causa mais comum de câncer em áreas com baixo IDH e a causa mais comum de morte por câncer em vários países com IDH baixo e médio (WÜNSCH-FILHO, 2008). Por exemplo, as taxas de incidência de câncer de colo de útero são maiores no leste, oeste e sul da África, região centro-sul da Ásia (principalmente Índia) e América do Sul (BRAY et al., 2012).

A baixa incidência de câncer de colo de útero em países desenvolvidos reflete o impacto do rastreamento em massa através do exame citológico do colo uterino. 0 objetivo do rastreamento é detectar, erradicar e acompanhar as lesões cervicais pré-invasivas, precursoras do câncer (HACKER, 2005).

Há uma grande diversidade no desenvolvimento socioeconômico e na assistência de saúde entre as diferentes regiões brasileiras e entre os vários municípios que compõem cada estado. Consequentemente, as taxas de mortalidade por câncer de mama e de colo de útero apresentam uma marcante heterogeneidade em todo o país (KLIGERMAN, 1999; JANUZZI, 2004; BABONES, 2008).

Com o aumento do número de mulheres chefes de família no Censo-2010 do IBGE e no Relatório de Desenvolvimento Humano-2010 do Programa das Nações Unidas para o Desenvolvimento (PNUD), podese prever que um maior número de famílias fica desprovido da pessoa responsável pela sua manutenção em consequência do aumento nas taxas de mortalidade por câncer em mulheres. Além disso, na sociedade brasileira, a mulher ainda é a principal responsável pelos cuidados com os filhos e com a família. Outros aspectos evidenciados pelo Censo-2010 são o processo crescente de urbanização da população e a inserção cada vez maior da mulher no mercado de trabalho (INSTITUTO BRASILEIRO DE GEOGRAFIA E ESTATISTICA, 2013).

A transição socioeconômica da população de baixa renda para média renda resulta na redução da prevalência de doenças infecciosas, e aumento daquelas relacionadas a fatores dietéticos, reprodutivos, metabólicos, hormonais e comportamentais. Por isso, há uma tendência de redução da incidência de câncer de colo de útero e aumento da incidência de câncer de mama no Brasil, principalmente nas cidades com menor desenvolvimento socioeconômico.

Um estudo ecológico sobre a mortalidade por câncer de mama de 1991 a 2010, nas cinco regiões brasileiras, mostrou que a tendência de mortalidade na região 
Sudeste é de queda e na região Sul é de estabilização, com os melhores Índices de Desenvolvimento Humano. Contrariamente, as regiões Centro-Oeste, Nordeste e Norte mostraram tendência de crescimento da mortalidade pelo câncer de mama e os piores Índices de Desenvolvimento Humano (KLUTHCOVSKY et al., 2014).

Um recente estudo sobre os contrastes na mortalidade por câncer de colo uterino e de mama nas capitais e demais municípios brasileiros, segundo indicadores socioeconômicos e assistenciais classificou os indicadores em positivos, correspondendo à melhor condição de vida, inclusive o IDH, e indicadores negativos, correspondendo à pior condição. Os coeficientes de mortalidade por câncer de colo de útero correlacionaram-se diretamente com os indicadores negativos e inversamente, com os positivos, nas capitais. No interior, somente o percentual de pessoas que vivem abaixo da linha da pobreza relacionou-se diretamente à mortalidade por este tipo de câncer. No caso do câncer de mama, as taxas de mortalidade correlacionaram-se diretamente com indicadores positivos e inversamente, com indicadores negativos, principalmente nos municípios do interior (GIRIANELLI; GAMARRA; SILVA, 2014).

As análises possuem limitações, especialmente porque elas são extremamente dependentes da disponibilidade e da acurácia dos dados. De uma forma geral, há uma noção geral de que os dados oriundos de locais com IDH alto e muito alto possuem maior qualidade que aqueles provenientes de locais com IDH médio e baixo. Os contrastes na ocorrência de câncer, finalmente, descrevem uma situação de saúde em que as discrepâncias na saúde tendem a aumentar de magnitude pelo processo de globalização e crescente urbanização. É possível, entretanto, observar estas discrepâncias em diferentes níveis de agregação, e os próximos capítulos farão esta análise distinta, qual seja: grandes regiões, estados e municípios.

\section{CONCLUSÃO}

Há um avanço geral dos estados brasileiros com relação aos indicadores sociais. Ao longo de 30 anos de observação, o câncer do colo do útero vem apresentando maior mortalidade nos estratos populacionais com pior desenvolvimento social. Em contraposição, o câncer de mama é mais expressivo nos estratos em que os indicadores podem ser considerados mais satisfatórios. Esta relação pode ser observada em diferentes níveis de agregação.

Estas evidências permitem discutir, portanto, a necessidade de mudanças na estratégia de operacionalização das políticas de atenção oncológica e de saúde da mulher. As prioridades da política de controle de câncer no Brasil baseiam-se no perfil de morbidade e mortalidade dos diversos estados 
e municípios do país, apresentando ampla variação de região para região. De posse das estimativas de casos incidentes e da mortalidade por câncer segundo localizações primárias, pode-se oferecer informações epidemiológicas que são fundamentais para 0 planejamento de ações de promoção à saúde, detecção precoce e de atenção oncológica em todos os níveis. Neste sentido, reconhecer as desigualdades regionais provocadas por diferenças no desenvolvimento contribui para descentralizar a pactuação das metas locais e de diversificar as estratégias de intervenção coletiva. É necessário, pois, melhorar o alcance das ações de rastreamento e diagnóstico precoce e, principalmente, acesso a tratamento, diante das desigualdades sociais do país, alcançando, assim, o princípio de equidade da política de saúde no Brasil.

\section{REFERÊNCIAS}

ALVES, C. M. M.; GUERRA, M. R.; BASTOS, R. R. Tendência de mortalidade por câncer de colo de útero para o Estado de Minas Gerais, brasil, 1980-2005. Cadernos de Saúde Pública, Rio de Janeiro, v. 25, n. 8, p. 16831700, ago. 2009.

AZEVEDO E SILVA, $G$ et al. Acesso à detecção precoce do câncer de mama no Sistema Único de Saúde: uma análise a partir dos dados do Sistema de Informações em Saúde. Cadernos de Saúde Pública, Rio de Janeiro, v. 30, n. 7, p. 1537-1550, jul. 2014.

BABONES, S. J. Income inequality and population health: Correlation and casuality.
Social Science and Medicine, [S. I.], v. 66, n. 7, p. 1614-26, apr. 2008.

BERNARD, V. B. et al. Vital signs: cervical cancer incidence, mortality, and screening United States, 2007-2012. Morbidity and Mortality Weekly Report, Atlanta, v. 63, p. 1-6, nov. 2014.. Disponível em: http://www.cdc.gov/mmwr, acesso em 7 junho de 2015.

BRASIL. Ministério da Saúde. Portaria ${ }^{0} 140$, de 27 de fevereiro de 2014. Redefine os critérios e parâmetros para organização, planejamento, monitoramento, controle e avaliação dos estabelecimentos de saúde habilitados na atenção especializada em oncologia e define as condições estruturais, de funcionamento e de recursos humanos para a habilitação destes estabelecimentos no âmbito do Sistema Único de Saúde (SUS). Brasília, DF, $2014 . \quad$ Disponivel em:<http://bvsms.saude.gov.br/bvs/saudelegis/ sas/2014/prt0140_27_02_2014.html>. Acesso em: 26 nov. 2015.

Departamento de Informática do SUS. Sistema de Informações sobre Mortalidade. Brasília, DF, 2015. Disponível em:<http://tabnet.datasus.gov.br/cgi/deftohtm.e xe?sim/cnv/obt10uf.def>. Acesso em: 2 jan. 2016.

BRAY, F. et al. Global cancer transitions according to the Human Development Index (2008-2030): a population-based study. Lancet, [S. I.], v. 13, n. 8, p.1-11, aug. 2012.

CLEGG, L. X.; REICHMAN, M. E.; MILLER, B. A. Impact of socioeconomic status on cancer incidence and stage at diagnosis: selected findings from the surveillance, epidemiology, and end results: National Longitudinal Mortality Study. Cancer Causes Control, [S. I.], v. 20, n. 4, p.417-435, mayo 2009.

DOLL, R.; PAYNE, P.; WATERHOUSE, J. Cancer incidence in five continents: a technical report. Berlin: Springer-Verlarg, 1966. 
FARMER, P.; FRENK, J.; KNAUL, F. M. Expansion of cancer care and control in countries of low and middle incomee: a call to action. The Lancet, [S. I.], v. 376, n. 9747, p. 1186-1193, oct. 2010.

FELIX, J. D. et al. Tendência de Mortalidade por Câncer de Mama em Mulheres no Estado do Espírito Santo, no período de 1980 a 2007. Revista Brasileira de Cancerologia, Rio de Janeiro, v. 57, n. 2, p. 159-166, 2011.

FERLAY, J. et al. Cancer incidence and mortality worldwide: Sources, methods and major patterns in GLOBOCAN 2012. International Journal of Cancer, [S. I.], v. 136, n. 5, p. E359-E386, mar. 2015.

et al. Estimates of worldwide burden of cancer in 2008: GLOBOCAN 2008. International Journal of Cancer, [S. I.], v. 127, n. 12, p. 2893-2917, dec. 2010.

GIRIANELLI, V. R.; GAMARRA, C. J.; SILVA, G. A. Os grandes contrastes na mortalidade por câncer do colo uterino e de mama no Brasil. Revista de Saúde pública, São Paulo, v. 48, n. 3, p. 459-467, 2014.

HACKER, N. F. Cervical Cancer. In: BEREK, J. S.; HACKER, N. F. Practical Gynecologic Oncology. 4a. ed. Philadelphia: Lippincott Williams 7 Wilkins, 2005. Cap. 9, p. 337-395.

INSTITUTO BRASILEIRO DE GEOGRAFIA E ESTATISTICA. Atlas do Censo Demográfico 2010. Riode Janeiro, 2013. Disponível em:<http://censo2010.ibge.gov.br/apps/atlas/>. Acesso em: 30 nov. 2014.

Estimativas populacionais para os municípios e Unidades da Federação brasileiros. Rio de Janeiro, 2015. Disponível em:<http://www.ibge.gov.br/home/estatistica/po pulacao/estimativa2015/default.shtm>. Acesso em: 2 jan. 2016.

INSTITUTO NACIONAL DE CÂNCER. Atlas de Mortalidade. Rio de Janeiro, 2015. Disponível em:<https://mortalidade.inca.gov.br>. Acesso em: 30 dez. 2015.

Tratamento do Câncer de mama: rotinas internas do INCa. 1. ed. Rio de Janeiro, 2010.

JANNUZZI PM. Indicadores sociais no Brasil: conceito, fontes de dados e aplicações. 3 ed. Campinas: Alínea, 2004.

JEMAL, A. et al. Global patterns of cancer incidence and mortality rates. Cancer Epidemiology, Biomarkers and Prevention, Philadelphia, PA, v. 19, n. 8, p. 1893-1907, aug. 2010.

et al. Global cancer statistics. CA: Cancer Journal for Clinicians, [S. I.], v. 61, n. 2, p. 69-90, mar./apr. 2011.

KATALINIC, A.; PRITZKULEIT, R.; WALDMANN, A. Recent trends in breast cancer incidence and mortality in Germany. Breast Care, Freiburg, v. 4, n. 2, p. 75-80, may 2009.

KLIGERMAN J. O câncer como um indicador de saúde no Brasil. Revista Brasileira de Cancerologia, Rio de Janeiro, v. 45, n. 3, p. 56, jul./ago./set. 1999.

KLUTHCOVSKY, A. C. G. C. et al. Female breast cancer mortality in Brazil and its regions. Revista da Associação Médica Brasileira, São Paulo, v. 60, n. 4, p. 387-393, 2014.

LAGES, RB et al. Desigualdades associadas à não realização de mamografia na zona urbana de Teresina-Piauí-Brasil,2010-2011. Revista Brasileira de Epidemiologia, São Paulo, v. 15, n. 4, p. 737-747743-747, 2012.

MOLINIÉ, F. et al. Trends in breast cancer incidence and mortality in France 1990-2008. Breast Cancer Research and Treatment, [S. I.], v. 147, n. 1, p. 167-175, aug. 2014.

REZENDE, M. C. R. et al. Causas do retardo na confirmação diagnóstica de lesões mamárias em mulheres atendidas em um 
centro de referência do Sistema Único de Saúde. Revista Brasileira de Ginecologia e obstetrícia, Rio de janeiro, v. 31, n. 2, p. 75-81, 2009.

RODRIGUES, A. D.; BUSTAMANTETEIXEIRA, M. T. Mortalidade por câncer de mama e câncer de colo de útero em município de porte médio da Região Sudeste do Brasil,1980-2006. Caderno de Saúde Pública, Rio de Janeiro, v. 27, n. 2, p. 241-248, fev. 2011.

SEGI, M. et al. The age-adjusted death rates for malignant neoplasms in some selected sites in 23 countries in 1954-1955 and their geographical correlation. The Tohoku Journal of Experimental Medicine, Japan, v. 72, n. 1, p. 91-103, 1960.

SILVA, G. A. et al. Tendência da mortalidade por câncer nas capitais e interior do Brasil entre 1980 e 2006. Revista de Saúde Pública, São Paulo, v. 45, n. 6, p. 1009-1018, dez. 2011.

THULER, L. C. S.; MENDONÇA, G. A. Estadiamento inicial dos casos de câncer de mama e de colo do útero em mulheres brasileiras. Revista Brasileira de Ginecologia e Obstetrícia, Rio de Janeiro, v. 27, n. 11, p. 655-60, 2005.

TORIOLA, A. T.; COLDITZ, G. A. Trends in breast cancer incidence and mortality in the United States: implications for prevention. Breast Cancer Research and Treatment, [S. I.], v. 138, n. 3, p. 665-673, apr. 2013.

WARD, E. et al. Cancer disparities by race/ethnicity and socioeconomic status. CA: Cancer Journal for Clinicians, [S. I.], v. 54, n. 2, p. 78-93, mar. 2004.

WÜNSCH-FILHO, V. et al. Perspectivas da investigação sobre determinantes sociais em câncer. Physis: Revista de Saúde Coletiva, Rio de Janeiro, v. 18, n. 3, p. 427-50, sept. 2008.
Doutor em Saúde Pública pelo Instituto de Estudos em Saúde Coletiva (IESC) da Universidade Federal do Rio de Janeiro (/UFRJ)

Pesquisador em Saúde Pública da Fundação Oswaldo Cruz (FIOCRUZ)

E-mail: raphael.guimaraes@fiocruz.br

\section{Camila Drumond Muzi}

Enfermeira

Mestre em Ciências pela Escola Nacional de Saúde

Pública Sergio Arouca (ENSP) da Fundação Oswaldo

Cruz (FIOCRUZ)

Tecnologista Pleno do Instituto Nacional de Câncer (INCA)

E-mail: camilamuzi@gmail.com

\section{Moema de Poli Teixeira \\ Cientista Social \\ Doutora em Antropologia pela Universidade Federal do Rio de Janeiro (UFRJ) \\ Assessora plena da Fundação Cesgranrio \\ E-mail: moemapoli@gmail.com}

\section{Sonoe Sugahara Pinheiro}

Engenheira

Doutora em Economia da Indústria e Tecnologia pela Universidade Federal do Rio de Janeiro (UFRJ)

Pesquisadora do Instituto Brasileiro de Geografia e

Estatística (IBGE)

E-mail: sonoe.pinheiro@ibge.gov.br

Fundação Oswaldo Cruz - FIOCRUZ

Av. Brasil, 4365 - Manguinhos, Rio de Janeiro/RJ CEP: $21040-900$

\section{Instituto Nacional de Câncer - INCA}

Praça Cruz Vermelha, 23, Centro, Rio de Janeiro/RJ CEP: 20230-130

\section{Fundação Cesgranrio}

Rua Santa Alexandrina, 1011, Rio Comprido, Rio de Janeiro/RJ

CEP: 20261-903

Instituto Brasileiro de Geografia e Estatística - IBGE Av. Franklin Roosevelt, $166 / 10^{\circ}$ andar, Castelo, Rio de Janeiro/RJ

CEP: 20021-120

\section{Raphael Mendonça Guimarães Enfermeiro}

R. Pol. Públ., São Luís, v. 20, n 1, p. 33-50, jan./jun. 2016. 